\title{
Digital Collections Are a Sprint, Not a \\ Marathon: Adapting Scrum Project \\ Management Techniques to Library \\ Digital Initiatives
}

Michael J. Dulock

and Holley Long

\begin{abstract}
This article describes a case study in which a small team from the digital initiatives group and metadata services department at the University of Colorado Boulder (CU-Boulder) Libraries conducted a pilot of the Scrum project management framework. The pilot team organized digital initiatives work into short, fixed intervals called sprints-a key component of Scrum. Working for more than a year in the modified framework yielded significant improvements to digital collection work, including increased production of digital objects and surrogate records, accelerated publication of digital collections, and an increase in the number of concurrent projects. Adoption of sprints has improved communication and cooperation between participants, reinforced teamwork, and enhanced their ability to adapt to shifting priorities.
\end{abstract}

\section{INTRODUCTION}

Libraries in recent years have freely adapted methodologies from other disciplines in an effort to improve library services. For example, librarians have

- employed usability testing techniques to enhance users' experience with digital libraries interfaces, ${ }^{1}$ improve the utility of library websites, ${ }^{2}$ and determine the efficacy of a visual search interface for a commercial library database; ${ }^{3}$

- adopted participatory design methods to identify information visualizations that could augment digital library services ${ }^{4}$ and determine user needs in new library buildings; ${ }^{5}$ and

- utilized principles of continuous process improvement to enhance workflows for book acquisition and implementation of serial title changes in a technical services unit. ${ }^{6}$

Librarians often come to the profession with disciplinary knowledge from an undergraduate degree unrelated to librarianship, so it should come as no surprise that they bring some of that disciplinary knowledge to their work. The interdisciplinary nature of librarianship also creates an environment that is amenable to adoption or adaptation of techniques from a variety of sources, not only those originating in library science. In this paper, the authors describe their experiences

Michael J. Dulock (michael.dulock@colorado.edu) is Assistant Professor and Metadata Librarian, University of Colorado Boulder. Holley Long (longh@uncw.edu), previously Assistant Professor and Systems Librarian for Digital Initiatives at University of Colorado, Boulder, is Digital Initiatives Librarian, Randall Library, University of North Carolina Wilmington. 
in applying a modified Scrum management framework to facilitate digital collection production. They begin by elucidating the fundamentals of Scrum and then describes a pilot project using aspects of the methodology. They discuss the outcomes of the pilot and posit additional features of Scrum that may be adopted in the future.

\section{Fundamentals of Scrum Project Management}

The Scrum project management framework-one of several techniques under the rubric of agile project management-originated in software development, and has been applied in a variety of library contexts including the development of digital library platforms ${ }^{7}$ and library web applications. ${ }^{8}$ Scrum's salient characteristics include self-managing teams that organize their work into "short iterations of clearly defined deliverables" and focus on "communication over documentation." "The Scrum Primer: A Lightweight Guide to the Theory and Practice of Scrum describes the roles, tools, and processes involved in this project management technique. ${ }^{10}$ Scrum teams are cross-functional and consist of five to nine members who are cross-trained to perform multiple tasks. In addition to the team, two individuals serve specialized roles, Scrum Master and Product Owner. The Scrum Master is responsible for ensuring that Scrum principles are followed and for removing any obstacles that hinder the team's productivity. Hence the Scrum Master is not a project manager, but a facilitator. The Product Owner's role is to manage the product by identifying and prioritizing its features. This individual represents the stakeholders' interests and is ultimately responsible for the product's value.

The team divides their work into short, fixed intervals called sprints that typically last two to four weeks and are never extended. At the beginning of each sprint, the team meets to select and commit to completing a set of deliverables. Once these goals are set, they remain stable for the duration; course corrections can occur in later sprints. In software development, the Scrum team aims to complete a unit of work that stands on its own and is fully functional, known as a potentially shippable increment. It is selected from an itemized list of product features called the product backlog. The backlog is established at the outset of development and consists of a comprehensive list of tasks that must occur to complete the product. A well-constructed backlog has four characteristics. First, it is prioritized with the features that will yield the highest return on investment at the top of the list. Second, the backlog is appropriately detailed, so that the tasks at the top of the list are well-defined whereas those at the bottom may be more vaguely demarcated. Third, each task receives an estimation for the amount of effort required to complete it, which helps the team to project a timeline for the product. Finally, the backlog evolves in response to new developments. Individual tasks may be added, deleted, divided, or reprioritized over the life of the project.

During the course of a sprint, team members meet to plan the sprint, check-in on a daily basis, and then debrief at the conclusion of the sprint. They begin with a two-part planning meeting in which the Product Owner reviews the highest priority tasks with the team. In the second half of the meeting, the team and the Scrum Master determine how many of the tasks can be accomplished in 
the given timeframe, thus defining the goals for the sprint. This meeting generally lasts no longer than four hours for a two-week sprint. Every day, the team holds a brief meeting to get organized and stay on track. During these "daily Scrums," each team member shares three pieces of information: what has been accomplished since the previous meeting, what will be accomplished before the next meeting, and what, if any, obstacles are impeding the work. These fifteen-minute meetings provide the team with a valuable opportunity to communicate and coordinate their efforts. Sprints conclude with two meetings, a review and retrospective. During the review, the team inspects the deliverables that were produced during that sprint. The retrospective provides an opportunity to discuss the process, what is working well, and what needs to be adjusted.

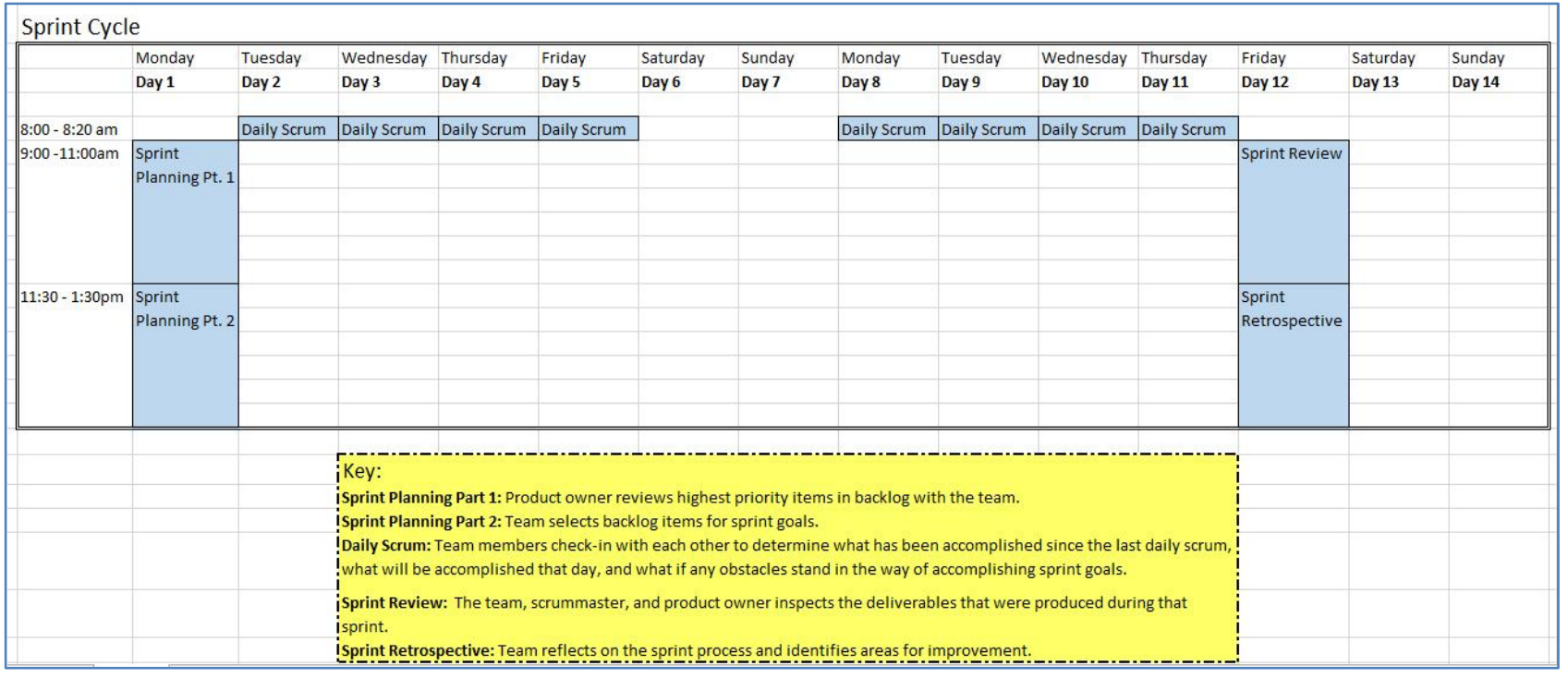

Figure 1. Typical Meeting Schedule for a Two-Week Sprint

Evidence in the literature suggests that Scrum improves both outcomes and process. One metaanalysis of 274 programming case studies found that implementing Scrum led to improved productivity as well as greater customer satisfaction, product quality, team motivation, and cost reduction. ${ }^{11}$ Proponents of this project management technique find that it leads to a more flexible and efficient process. Scrum's brief iterative work cycles and evolving product backlog promote adaptability so the team can address the inevitable changes that occur over the life of a project. By contrast, traditional project management techniques have been criticized for requiring too much time upfront on planning and being too rigid to respond to changes in later stages of the project. ${ }^{12}$ Scrum also promotes communication over documentation, ${ }^{13}$ resulting in less administrative overhead as well as increased accountability and trust between team members.

\section{Scrum Pilot at University of Colorado Boulder Libraries}

The University of Colorado Boulder (CU-Boulder) Libraries digital initiatives team was interested in adopting Scrum because of its incremental approach to completing large projects, its focus on communication, and its flexibility. These attributes meshed well with the group's goals to publish larger collections more quickly and to more effectively multitask the production of multiple high 
priority collections. The group's staffing model and approach to collection building prior to the Scrum pilot is described here to provide some context for this choice of project management tool.

Digital collection proposals are vetted by a working group composed of ten members, the Digital Library Management Group (DLMG), to ensure that major considerations such as copyright status are fully investigated before undertaking the collection. Approved proposals are prioritized by the appropriate collection manager as high, medium, or low and then placed in a queue for scanning and metadata provisioning. A core group of individuals generally works on all digital collections, including the metadata librarian, the digital initiatives librarian, and one or both of the digitization lab managers. Additionally, the team frequently includes the subject specialist who nominated the collection for digitization, staff catalogers, and other library staff members whose expertise is required. At any given time, the queue may contain as many as fifteen collections, and the core team works on several of them concurrently to address the separate needs of participating departments. While this approach allows the teams to distribute resources more equitably across departments, progress on individual collections can be slower than if they are addressed one at a time. Prior to implementing aspects of Scrum, the team also completed the scanning and metadata records for every object in the collection before it was published. As a result, publication of larger collections trailed behind smaller collections.

The details of digital collection production vary depending of the nature of the project, but the process usually follows the same broad outline. Unless the entire collection will be digitized, the collection manager chooses a selection of materials on the basis of criteria such as research value, rarity, curatorial considerations, copyright status, physical condition, feasibility for scanning, and availability of metadata. Photographs and paper-based materials are then evaluated by the preservation department to ensure that they are in suitable condition for scanning. Likewise, the media lab manager evaluates audio and video media for condition issues such as sticky shed syndrome, which will affect digitization. ${ }^{14}$ Depending on format, the material is then digitized by the digitization lab manager or the media lab manager and their student assistants according to locally established workflows that conform to nationally recognized best practices. Once digitized, student assistants apply post-processing procedures as appropriate and required, such as running OCR (optical character recognition) software to convert images to text or equalizing levels on an audio file. The lab managers then check the files for quality assurance and move the files to the appropriate location on the server. The metadata librarian creates a metadata template appropriate to the material being digitized by using industry standards such as Visual Resources Association Core (VRA Core), Metadata Object Description Schema (MODS), PBCore, and Dublin Core (DC). Metadata creation methods depend on the existence of legacy metadata for the analog materials and in what format legacy metadata is contained. The metadata librarian, along with his staff and/or student assistants, adapts legacy metadata into a format that can be ingested by the digital library software or creates records directly in the software when there is no legacy metadata. Metadata is formatted or created in accordance with existing input standards such as Cataloging Cultural Objects (CCO) and Resource Description and Access (RDA), and it is enhanced 
as much as possible using controlled vocabularies such as the Art and Architectural Thesaurus (AAT) and Library of Congress Subject Headings. The metadata librarian performs quality assurance on the metadata records during creation and before the collection is published. In the final stages, the collection is created in the digital library software, at which time search and display options are established: thumbnail labels, default collection sorting, faceted browsing fields, etc. Then the files and metadata are uploaded and published online.

The highlight of the CU-Boulder Digital Library is the twenty-seven collections drawn from local holdings in Archives, Special Collections Department, Music Library, and Earth Sciences and Map Library, among others. The Library also contains purchased content and "LUNA Commons" collections created by institutions that use the same digital library platform, for a total of more than 185,000 images, texts, maps, audio recordings, and videos. The following four collections were created during the Scrum pilot and illustrate the types of materials available in the CUBoulder Digital Library:

\begin{tabular}{|l|l|}
\hline & $\begin{array}{l}\text { The Colorado Coal Project consists of video and audio } \\
\text { interviews, transcripts, and slides collected between 1974 and } \\
1979 \text { by the University of Colorado Coal Project. The project } \\
\text { was funded by the Colorado Humanities Program and the } \\
\text { National Endowment for the Humanities to create an } \\
\text { ethnographic record of the history of coal mining in the } \\
\text { western United States from immigration and daily life in the } \\
\text { coal camps to labor conditions and strikes, including Ludlow } \\
(1913-14) \text { and Columbine (1927). }\end{array}$ \\
\hline
\end{tabular}

The Mining Maps Collection provides access to scanned maps of various mines, lodes, and claims in Colorado from the late 1800 s to the early 1900s. These maps come from a variety of creators, including private publishers and US government agencies. 


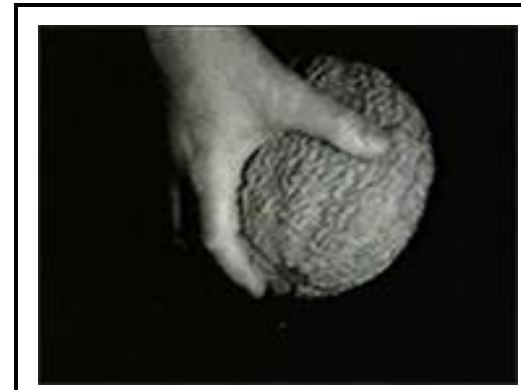

The Vasulka Media Archive showcases the work of pioneering video artists Steina and Woody Vasulka and contains some of their cutting-edge studies in video that experiment with form, content, and presentation. Steina, an Icelander, educated in music at the Prague Conservatory of Music, and Woody, a graduate of Prague's Film Academy, arrived in New York City just in time for the new media explosion. They brought with them their experience of the European media awakening, which helped them blend seamlessly into the youth media revolution of the late sixties and early seventies in the United States.

The 3D Natural History Collection comprises one hundred archaeology and paleontology specimens from the Rocky Mountain and Southwest regions, including baskets, moccasins, animal figurines, game pieces, jewelry, tools, and other everyday objects from the Freemont, Clovis, and Ancestral Puebloan cultures as well as a selection of vertebrate, invertebrate, and track paleontology specimens from the Mesozoic through the Cenozoic Eras (250 Ma to the present).

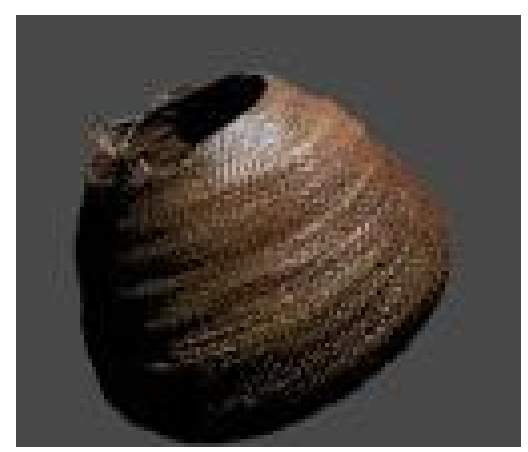

The diffusion of effort across multiple collections and a slower publication rate for larger collections offered opportunities for improvement. After attending a conference session on Scrum project management for web development projects, one of the team members recognized Scrum's potential to improve production processes since the technique divides large projects into manageable subtasks that can be accomplished in regular, short intervals. ${ }^{15}$ This approach would allow the team to switch between different high priority collections at regularly defined intervals to facilitate steady progress on competing priorities. Working in sprints would also make it easier to publish smaller portions of a large collection at regular intervals. Thus Scrum held the potential to increase the production rate for larger collections and make the team's progress more transparent to users and colleagues.

In April 2013, a small team of CU-Boulder librarians and staff initiated a pilot to assess the effect on processes and outcomes for digital collection production. Rather than involving individuals from all affected units, regardless of their level of engagement in a particular project, the Scrum pilot was limited to the three individuals who were involved in most, if not all, of the projects 
undertaken: the digital initiatives librarian, metadata librarian, and digitization lab manager. ${ }^{16} \mathrm{By}$ including these three individuals, the major functions of metadata provision, digitization, and publication were covered in the trial with no disruption to the existing workflows or organizational structures. Selecting this group also ensured that Scrum would be tested in a broad range of scenarios and on collections from several different departments.

To begin, the team met to review the Scrum project management framework and considered how best to pilot the technique. Taking a pragmatic approach, they only adopted those aspects of Scrum that were deemed most likely to result in improved outcomes. If the pilot were successful, other aspects of Scrum could be incrementally incorporated later. The group discussed how Scrum roles, processes, and tools could be adapted to digital collection workflows and determined that sprints would likely have the highest return on investment. They also chose to adapt and hybridize certain aspects of the planning meeting and daily scrum to achieve goals that were not being met by other existing meetings.

Sprint planning and end meetings were combined so that all three participants knew what each had completed and what was targeted for the next sprint. Select activities of sprint planning and end meetings were already a part of the monthly DLMG meetings, making additional sprint meetings redundant. Daily Scrum meetings were excluded as the team felt that daily meetings would not produce enough benefit to justify the costs. In addition, two of the three participants have numerous responsibilities that lie outside of projects subject to the Scrum pilot, so each person does not necessarily perform Scrum-related work every day. However, the short meeting time was adopted into the planning/end meeting, as were elements of the three core questions of the daily Scrum meeting, with some modifications. The questions addressed in the biweekly meetings are: What have you done since the last meeting? What are you planning for the next meeting? What impediments, if any, did you encounter during the sprint? The latter question was sometimes addressed mid-sprint through emails, phone calls, or one-off meetings that include a larger or different group of stakeholders.

The team adopted the two-week duration typical of Scrum sprints for the pilot. This has proven to be a good medium-term timeframe. It was short enough that the team could adjust priorities quickly, but long enough to complete significant work. The team chose to combine the sprint planning and sprint review meetings into a single meeting. Part of the motivation for a trial of the Scrum technique was to minimize additional time away from projects while maximizing information transfer during the meetings. A single biweekly planning/review meeting was determined to be sufficient to report accomplishments and set goals yet substantial and free of irrelevant content without being overly burdensome as "yet another meeting."

At each sprint meeting, each participant reported on results from the previous sprint. Work that was completed allowed the next phase of a project to proceed. Based on the results of the last sprint, each team member set measurable goals that could be realistically met in the next twoweek sprint. There has been a concerted effort to keep the meetings short, limited to about twenty 
to twenty-five minutes. To enforce this habit, the sprint meetings were scheduled to begin twenty minutes before other regularly scheduled meetings for most or all of the participants. This helped keep participants on-topic and reinforced the transfer-of-information aspect of the meetings, with minimal leeway for extraneous topics.

\section{REFLECTION}

The modified Scrum methodology described above has been in place for more than a year. There have been several positive outcomes resulting from this practice. Beginning with the most practical, production has become more regular than it was before Scrum was implemented. The nature of digital initiatives in this environment dictates that many projects are in progress at once, in various stages of completion. The production work, such as digitizing media or creating metadata records, has become more consistent and regular. Instead of production peaks and valleys, there is more of a straight line as portions of projects are finished and others come online. This in turn has resulted in faster publication of collections. In 2013, the team published six new collections, twice as many as the previous year. The ability to put all hands on deck for a project for a two-week period can increase productivity. Since sprints allow for short, concentrated bursts of work on a single project, smaller projects can be completed in a few sprints and larger projects can be divided into "potentially shippable units" and thus published incrementally.

Another benefit of Scrum is that the variability of the two-week sprint cycle allows the team to work on more collections concurrently. For example, during a given sprint, scanning is underway for one collection, a metadata template is being constructed for another, the analog material in a third is being examined for pre-scanning preservation assessment, and a fourth collection is being published. While this type of multitasking occurred before the team piloted sprints, the Scrum project management framework lends more structure and coordination to the various team members' efforts. Collection building activities can be broken down into subtasks that are accomplished in nonconsecutive sprints without undercutting the team's concerted efforts. As a result, the team can juggle competing priorities much more effectively. The team is working with multiple stakeholders at any given time, each of whom may have several projects planned or in progress. As focus shifts among stakeholders and their respective projects, the Scrum team is able to adjust quickly to align with those priorities, even if only for a single sprint. This also makes it easier to respond to emerging requests or address small, focused projects on the basis of events such as exhibits or course assignments.

Additional benefits of the Scrum methodology pertain to communication and work style among the three Scrum participants. The frequent, short meetings are densely packed and highly focused. Each person has only a few minutes to describe what has been accomplished, explain problems encountered, troubleshoot solutions, and share plans for the next sprint. The return on the time investment of twenty minutes every two weeks is significant-there is no time to waste on issues that do not pertain directly to the projects underway, just completed, or about to start. A further result is that the group's sense of itself as a team is enhanced. As stated above, the three Scrum 
participants do not all work in the same administrative unit within the library. Though they shared frequent communication by email as projects progressed, regular sprint meetings have fostered a closer sense of team. The participants know from sprint to sprint what the others are doing; they can assist one another with problems face-to-face and coordinate with one another so that work segments progress toward production in a logical sequence.

With more than a year of experience with Scrum, the pilot team has determined that several aspects of the methodology have worked well in our environment. In general, the sprint pattern fits well with existing operating modes. The monthly DLMG meeting, which includes a large and diverse group, provides an opportunity to discuss priorities, review project proposals, establish standards, and make strategic decisions. The bi-weekly sprint meetings dovetail nicely, with one meeting taking place at a midpoint between DLMG meetings, and one just prior to DLMG meetings. This allows the three Scrum participants to focus on strategic items during the DLMG meeting but keep a close eye on operational items in between.

The Scrum methodology has also accommodated the competing priorities that the three participants must balance on an ongoing basis. There is considerable variation between participants in terms of roles and responsibilities, but the division of work into sprints has given the team greater opportunity to fit production work in with other responsibilities, such as supervision and training; scholarly research and writing; service performed for disciplinary organizations; infrastructure building; and planning, research, and design work for future projects. The two-week sprint duration is a productive time interval during which the team can set and reach incremental goals, whether that is starting and finishing a small project on short notice, making a big push on a large-scale project, or continuing gradual progress on a large, deliberatelypaced initiative. The brief meetings ensure that participants focus on the previous sprint and the upcoming sprint. There is usually just enough time to discuss accomplishments, goals, and obstacles, with some time left to troubleshoot as necessary. The meeting schedule and structure allows each individual to set his or her own goals so that he or she can make maximum progress during the sprint. This in turn feeds into accountability. There is always an external check on one's progress - the next meeting comes up in two weeks, creating an effective deadline (which also sometimes corresponds to a project deadline). It becomes easier to stay on task and keep goals in sight with the sprint report looming in a matter of days. At the same time, Scrum helps to define each person's role and clarifies how roles align with each other. Some tasks are completely independent, while others must be done in sequence and depend on another's work. The sprint schedule allows large, complex projects to be divided into manageable pieces so that each sprint can result in a sense of accomplishment, even if it may require many sprint cycles to actually complete a project. This is especially true for large digital initiatives. For instance, completing the entire project may take a year, but subsets of a collection may be published in phases at more frequent intervals in the meantime. 
Summary of Benefits

- Enhanced ability to manage multiple concurrent projects

- Published large collections incrementally, increasing responsiveness to users and other stakeholders

- Improved team building

- Increased communication and accountability among team members

\section{FUTURE CONSIDERATIONS}

Based on these outcomes, the team can safely say that it met its objectives for the test pilot. One of the reasons that it was feasible to try this when the participants were already highly committed is that the pilot used a small portion of the Scrum methodology and was not too rigid in its approach. The team felt that a hybrid of the Scrum planning and Scrum review meeting held twice a month would provide the benefits without overburdening schedules with additional meetings. There were also plans to have a virtual email check-in every other week to loosely achieve the goals of the daily Scrum meeting, that is, to improve communication and accountability. The email check-in fell by the wayside; the team found it wasn't necessary because there were already adequate opportunities to check-in with each other over the course of a two-week sprint.

The team has found the sprints and modified Scrum meetings to be highly useful and relatively easy to incorporate into their workflows. The next phase of the pilot will implement product backlogs and burn down charts, diagrams showing how much work remains for the team in a single sprint, with the goal of tracking collections' progress at the item level through each step of the planning, selection, preservation assessment, digitization, metadata provisioning, and publication workflows.

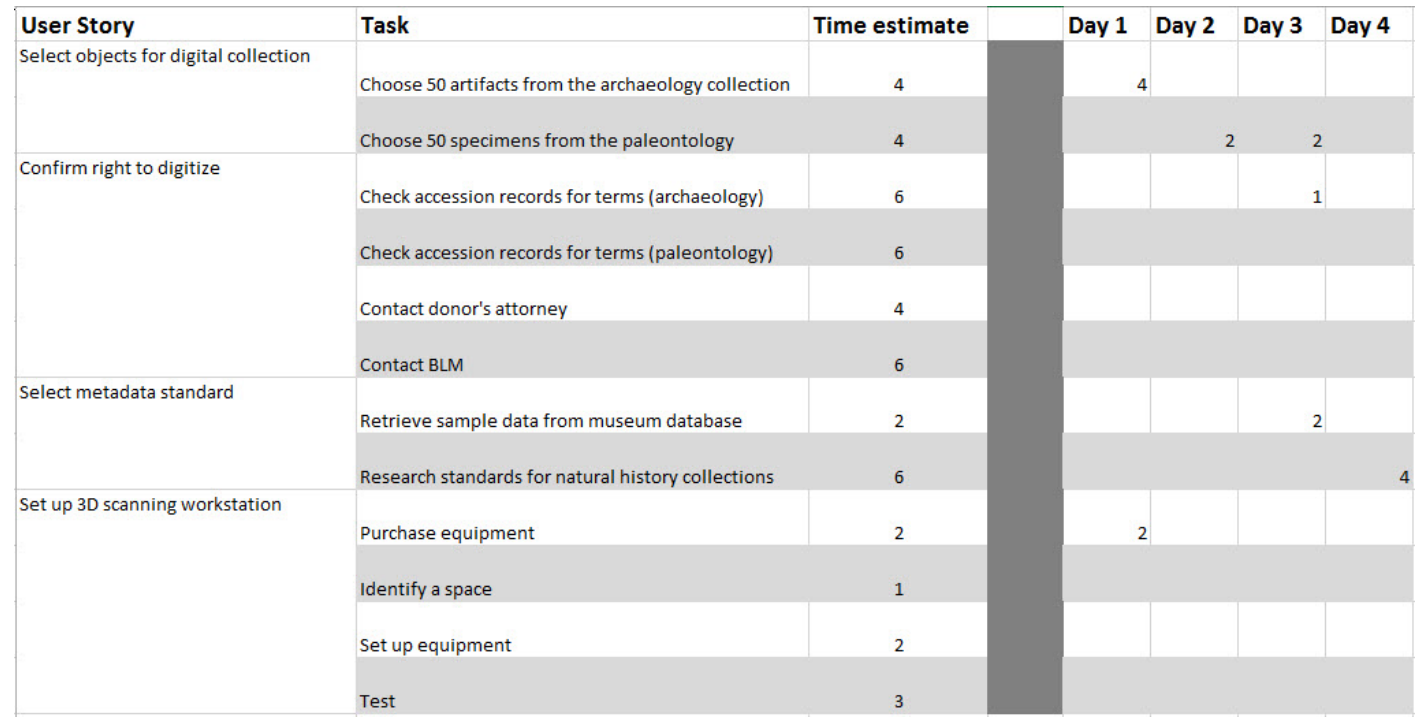

Figure 2. Hypothetical Backlog for the First Sprint of a Digital Collection ${ }^{17}$ 
Scrum backlogs are arranged on the basis of a task's perceived benefit for customers. To adapt backlogs for digital collection production work, the backlog task list's order will instead be based in part on the workflow sequence. For example, pieces from the physical collection must be selected before preservation staff can assess them. Additionally, the backlog items will be sequenced according to the materials' research value or complexity. For instance, the digitization of a folder of significant correspondence from an archival collection would be assigned a higher priority in the backlog than the digitization of newspaper clippings of minor importance from the same collection. Or, materials that are easy to scan would be listed in the backlog ahead of fragile or complex items that require more time to complete. This will allow the team to publish the most valuable items from the collection more quickly. According to Scrum best practices, backlogs are also appropriately detailed. In the context of digital collection production work, collections' backlogs would begin with a standard template of high-level activities: materials' selection, copyright analysis, preservation assessment, digitization, metadata creation, and publication. As the team progresses through backlog items, they will become increasingly detailed. Backlogs also evolve. Scrum's ability to respond to change has been one of its strongest assets in this environment and therefore the backlog's ability to evolve will make it a valuable addition to the team's process. For example, materials that a collection manager uncovers and adds to the project late in the process can be easily incorporated into the backlog or materials in the collection that are needed to support an upcoming instruction session can be moved up in the backlog for the next sprint. In this way, the backlog will support the team's goal to nimbly respond to shifting priorities and emerging opportunities.

\section{Burn Down Chart: 3D Natural History Collection}

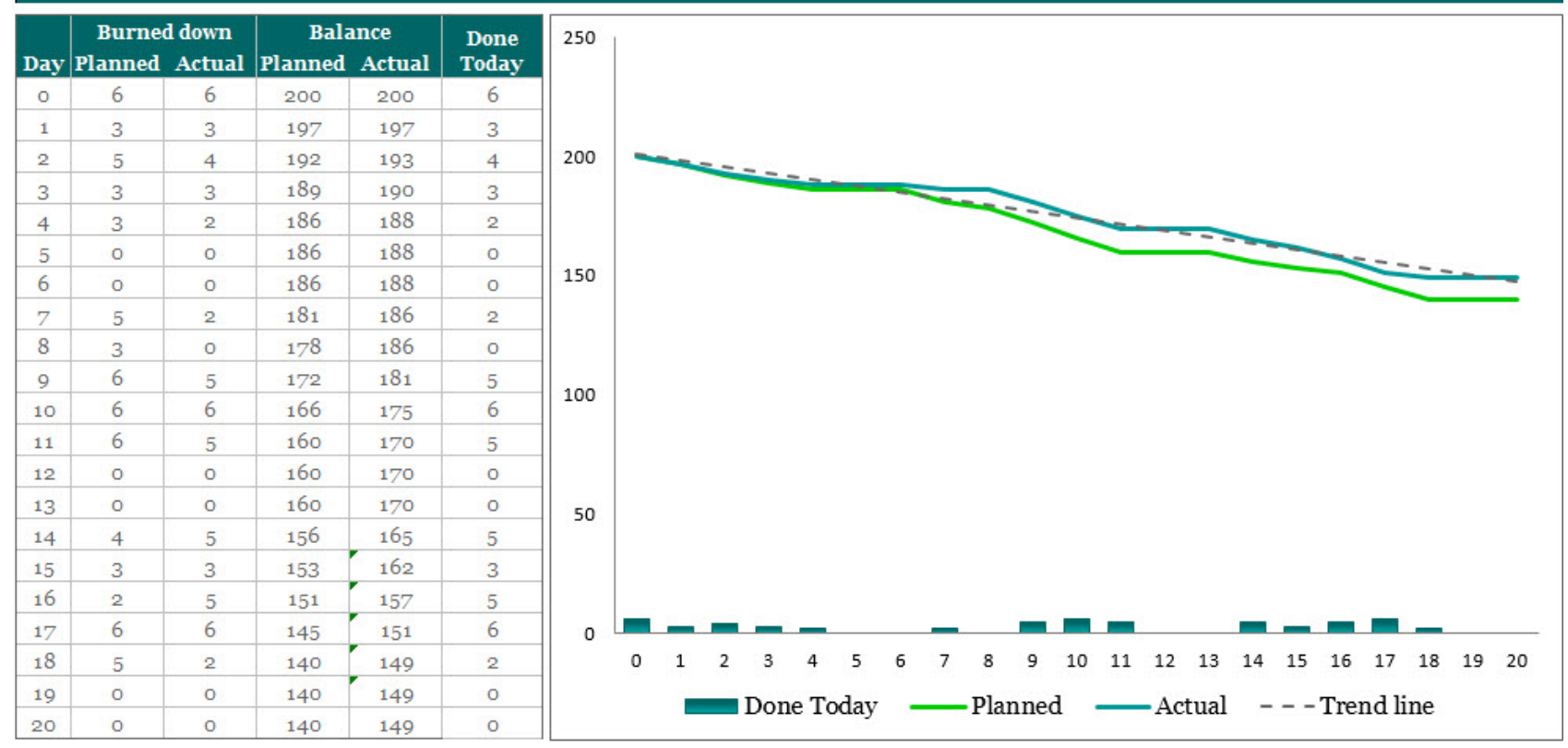

Figure 3. Hypothetical Burn Down Chart 18 
The final relevant feature of a backlog, the "effort estimates," taken in conjunction with the burn down chart will help the team develop better metrics for estimating the time and resources required to complete a collection. When items are added to the backlog, team members estimate the amount of effort needed to complete it. The burn down chart illustrates how much work remains and, in general practice, is updated on a daily basis. Given that the team has truncated the Scrum meeting schedule, this may occur on a weekly basis, but will nonetheless benefit the team in several ways. Initially, it will keep the team on track and provide valuable and detailed information for stakeholders on the collections' progress. As the team accrues old burn down charts from completed collections, they can use the data to hone their ability to estimate the amount of time and resources needed to complete a given project.

\section{CONCLUSION}

Through the pilot conducted for digital initiatives at CU-Boulder Libraries, application of aspects of the Scrum project management framework has demonstrated significant benefits with no discernable downside. Adoption of sprint planning and end meetings resulted in several positive outcomes for the participants. Digital collection production has become more regular; work can be underway on more collections simultaneously; and collections are, on average, published more quickly. In addition, communication and cooperation among the sprint pilot participants have increased and strengthened the sense of teamwork among them. The sprint schedule has blended well with existing digital initiatives meetings and workflows, and has enhanced the team's ability to handle ever-shifting priorities. Additional aspects of Scrum, such as product backlogs and burn down charts, will be incorporated into the participants' workflows to allow them to better track the work done at the item level, provide more detailed information for stakeholders during the course of a project, and predict how much time and effort will be required for future projects. The positive results of this pilot demonstrate the benefits to be gained by looking outside standard library practice and adopting techniques developed in another discipline. Given the range of activities performed in libraries, the possibilities to improve workflows and increase efficiency are limitless as long as those doing the work keep an open mind and a sharp eye out for methodologies that could ultimately benefit their work, and in turn, their users.

\section{REFERENCES}

1. Sueli Mara Ferreira and Denise Nunes Pithan, "Usability of Digital Libraries," OCLC Systems \& Services: International Digital Library Perspectives 21, no. 4 (2005): 316, doi: 10.1108/10650750510631695.

2. Danielle A. Becker and Lauren Yannotta, "Modeling a Library Web Site Redesign Process: Developing a User-Centered Web Site Through Usability Testing," Information Technology \& Libraries 32, no. 1 (2013): 11, doi: 10.6017/ital.v32i1.2311.

3. Jodi Condit Fagan, "Usability Testing of a Large, Multidisciplinary Library Database: Basic Search and Visual Search," Information Technology \& Libraries 25 no. 3 (2006): 140-41, 10.6017/ital.v25i3.3345. 
4. Panayiotis Zaphiris, Kulvinder Gill, Terry H.-Y. Ma, Stephanie Wilson and Helen Petrie, "Exploring the Use of Information Visualization for Digital Libraries," New Review of Information Networking 10, no. 1 (2004): 58, doi: 10.1080/1361457042000304136.

5. Benjamin Meunier and Olaf Eigenbrodt, "More Than Bricks and Mortar: Building a Community of Users through Library Design," Journal of Library Administration 54 no. 3 (2014): 218-19, 10.1080/01930826.2014.915166.

6. Lisa A. Palmer and Barbara C. Ingrassia, "Utilizing the Power of Continuous Process Improvement in Technical Services," Journal of Hospital Librarianship 5 no. 3 (2005): 94-95, 10.1300/J186v05n03 09.

7. Javier D. Fernández et al., "Agile DL: Building a DELOS-Conformed Digital Library Using Agile Software Development," in Research and Advanced Technology for Digital Libraries, edited by Birte Christensen-Dalsgaard et al. (Berlin: Springer-Verlag, 2008), 398-9, doi: 10.1007/978-3$\underline{540-87599-444}$.

8. Michelle Frisque, "Using Scrum to Streamline Web Applications Development and Improve Transparency" (paper presented at the 13th Annual LITA National Forum, Atlanta, Georgia, September 30-October 3, 2010).

9. Frank H. Cervone, "Understanding Agile Project Management Methods Using Scrum," OCLC Systems \& Services 27, no. 1 (2011): 19, 10.1108/10650751111106528.

10. Pete Deemer, Gabrielle Benefield, Craig Larman, and Bas Vodde, "The Scrum Primer: A Lightweight Guide to the Theory and Practice of Scrum," (2012), 3-15, www.infoq.com/minibooks/Scrum_Primer.

11. Eliza S. F. Cardozo et al., "SCRUM and Productivity in Software Projects: A Systematic Literature Review" (paper presented at the 14th International Conference on Evaluation and Assessment in Software Engineering (EASE), 2010), 3.

12. Cervone, "Understanding Agile Project Management," 18.

13. Ibid., 19.

14. Sticky shed syndrome refers to the degradation of magnetic tape where the binder separates from the carrier. The binder can then stick to the playback equipment rendering the tape unplayable.

15. Frisque, "Using Scrum."

16. The media lab manager responsible for audio and video digitization did not participate because his lab offers fee-based services to the public and thus has long-established business processes in place that would not have blended easily with sprints.

17. Figure 2 is based on illustration created by Mountain Goat Software, "Sprint Backlog," https://www.mountaingoatsoftware.com/agile/scrum/sprint-backlog.

18. Figure 3 is adapted from template created by Expert Project Management, "Burn Down Chart Template," www.expertprogrammanagement.com/wpcontent/uploads/templates/burndown.xls. 